\title{
A comparative study of anti-atherosclerotic/anti-inflammatory effect of irbesartan and rosuvastatin in hypercholesterolemic male rabbits
}

\author{
Murooj L Majeed, ${ }^{a}$ Hind F Mahdi, ${ }^{a}$ Najah R Hadi, ${ }^{a}$ and Ali M Janabib
}

\author{
aDepartment of Pharmacology and Therapeutic, Faculty of Medicine, University of Kufa, Najaf, Iraq. \\ bDepartment of Pharmacology and Toxicology, Faculty of Pharmacy, University of Kufa, Najaf, Iraq. \\ Correspondence to Najah R Hadi (email: drnajahhadi@yahoo.com). \\ (Submitted: 08 January 2019 - Revised version received: 15 February 2019 - Accepted: 12 March 2019 - Published online: 26 April 2019)
}

\begin{abstract}
Objective To evaluate the potential protective effect of irbesartan on atherosclerotic progression using rosuvastatin as a positive control. Methods Twenty local domestic rabbits were randomly divided into four groups, five rabbits per each group. The first group (I), rabbits were receiving normal diet; the second group (II), rabbits were receiving $2 \%$ cholesterol diet; the third group (III), rabbits were receiving rosuvastatin and $2 \%$ cholesterol diet and; the fourth group (IV), rabbit were receiving irbesartan and $2 \%$ cholesterol diet. Serum level of cholesterol, triglycerides, LDL, VLDL and HDL were determined after 12 weeks of the study. Serum level of high sensitive C-reactive protein, ICAM1 and VCAM1 were measured after 12 weeks of the study. Total antioxidant activity was also determined. Aortic tissue sent for histopathological examination of atherosclerosis lesion.

Results Data of this study have showed that atherogenic diet caused an increase in serum level of TC, LDL, VLDL and TG and a decrease in serum level of HDL. Treatment of rabbits with irbesartan caused substantial decrease in serum lipid profile compared with untreated rabbit receiving atherogenic diet. No significant difference was seen between irbesartan- and rosuvastatin-treated rabbits in all lipid parameters measured with the exception that the HDL level in rosuvastatin-treated rabbits was significantly higher than that of irbesartan-treated rabbits. Rabbit treated with irbesartan showed a significant decrease in the serum level of high sensitive C-reactive protein, ICAM1, VCAM1 and aortic total antioxidant capacity when compared with untreated group. No significant difference was seen between irbesartan- and rosuvastatin-treated rabbits in all these inflammatory parameters measured. Histopathological examination showed that irbesartan decreased atherogenic lesion significantly when compared with untreated rabbits.

Conclusion Irbesartan has a potential protective effect against atherosclerosis via decreasing lipid oxidation and inflammatory mediators. In terms of lipid lowering and anti-inflammatory effects, irbesartan has similar pattern of effectiveness to rosuvastatin.

Keywords anti-inflammatory, irbesartan, rosuvastatin calcium, hypercholesterolemic
\end{abstract}

\section{Introduction}

Atherosclerosis is a chronic inflammatory and immune disease that affects medium- and large-size arteries and begins with thickening in the wall of artery due to accumulation of monocytes, macrophages, T-lymphocytes, endothelial cells, smooth muscle cell and formation of fatty streak that form fibrous capsule with macrophage at the site of injury. ${ }^{1}$

Many risk factors are involved in atherosclerosis including fatty diet, obesity, diabetes hypercholesterolemia, hypertension, smoking. Some risk factors that related to establish the disease such as elevation of blood level of lipid and binding of oxidized lipid (LDL, VLDL) to the endothelium cell. ${ }^{2}$

The mechanistic way for formation of atherosclerosis lesion involves binding of monocyte and T-cell to the endothelium cell then migrate to the space of sub endothelium. Furthermore, monocyte oxidized and engulf LDL to form foam cell. That is important stage to generate proinflammatory cytokines and chemokines that recruit SMC and increase density of extracellular matrix ultimately to form atherosclerosis lesion. Moreover, this process stimulates inflammatory cell and platelet adherence that promote pro-atherogenic growth factor.

Inflammation is a crucial process responsible for progression of the disease. In acute phase of inflammation, liver releases an inflammatory protein, CRP and elevates both cholesterol and LDL which promote inflammatory mediator interleukin and monocyte chemoattractant protein 1 . This can stimulate expression of scavenger receptors responsible for accumulation of lipid and form fatty streak and foam cell. ${ }^{5}$
Rosuvastatin, is a statin that was used in treatment of hyperlipidemia, improves atherosclerosis by decrease pro-inflammatory cytokines and chemokines. ${ }^{6}$ On other hand, irbesartan, is an angiotensin receptor blocker used in treatment of hypertension, may improve atherosclerosis via alleviating the inflammatory response. ${ }^{5}$ The expression of ICAM1 in the vascular endothelium and its role in the early phase of atherosclerosis first includes inflammatory cell recruitment from circulation to the site of inflammation. Additionally, ICAM1 induces accumulation and adhesion of inflammatory cell on the surface of vascular endothelium. ${ }^{7}$ Another mediator, VCAM, promotes adhesion of inflammatory cell on vascular endothelium and facilitate movement of leukocyte along the surface of endothelium, and this adhesion promotes proliferation of vascular smooth muscles, so it is involved in atherosclerosis pathogenicity. ${ }^{8}$

The aim of this study is to further investigate potential athero-protective effect of irbesartan using rosuvastatin as a positive control.

\section{Materials and Methods}

About 20 local domestic rabbits (weigh 1.5-2 kg) were randomly classified into four groups. The first group (I), rabbits were receiving normal diet; the second group (II), rabbits were receiving $2 \%$ cholesterol diet; the third group (III), rabbits were receiving rosuvastatin $(2.5 \mathrm{mg} / \mathrm{kg} /$ day $)$ and $2 \%$ cholesterol diet and; the fourth group (IV), rabbit were receiving irbesartan $(10 \mathrm{mg} / \mathrm{kg} /$ day $)$ and $2 \%$ cholesterol diet. 
Distill water was used as vehicle for both rosuvastatin and Irbesartan. Drug treatments were given orally using gastric gavage. ${ }^{5,9,10}$ Animals were kept in controlled temperature and humidity as well as 12-h light/dark cycle in the animal house/ Faculty of Medicine/University of Kufa. Serum level of cholesterol, triglycerides, LDL, VLDL and HDL were determined after 12 weeks of the study. At the end of the study, animals were anesthetized with droperidol $(5 \mathrm{mg} / \mathrm{kg})$ and ketamine $(50 \mathrm{mg} / \mathrm{kg})$ before scarification. Serum level of high sensitive C-reactive protein (hs-CRP), ICAM1 and VCAM1 were measured after 12 weeks of the study. Total antioxidant activity was also determined at the end of the study. Aortic tissues were sent for histopathological examination of atherosclerosis lesion.

\section{Blood Sampling}

At 12 weeks after study, $3 \mathrm{ml}$ blood was obtained from the central ear artery of each overnight starved rabbit. Blood samples were left until clotted then centrifuged for $12 \mathrm{~min}$ at $6000 \mathrm{rpm}$ to obtain serum. Total lipid profile (TC, HDL, LDL, VLDL, TG) was measured. At the end of study, rabbits were anesthetized, and $5 \mathrm{ml}$ of blood was directly obtained from the heart to examine the serum level of hs-CRP, ICAM1 and VCAM1.

\section{Tissue Preparation for Measurement of Total Antioxidant Capacity}

Aortic tissue from each rabbit was taken and frozen down at $-80^{\circ} \mathrm{C}$. Samples were then homogenized using sonicate after washing with phosphate buffer saline. Triton $\mathrm{X}$ was then added to each sample, then sonicated in ice box and centrifuged for $30 \mathrm{~min}$ at $6000 \mathrm{rpm}$. Supernatant was obtained and analyzed for total antioxidant capacity. ${ }^{11}$

\section{Statistical Analyses}

All data are either representative or mean \pm SEM. Statistical comparisons were by one-way ANOVA followed by Bonferroni's test for multiple comparisons using Prism (GraphPad 7 Software Inc., San Diego, CA, USA). Statistical significance was considered for $P<0.05$.

\section{Results}

\section{Effect on Lipid Profile}

At the end of the study, serum cholesterol, triglyceride, LDL, VLDL levels were significantly increased, while serum HDL level was significantly decreased in cholesterol diet group when compared with normal diet control group (Fig. 1). However, serum cholesterol, HDL, TG, LDL, VLDL were significantly decreased in hyperlipidemic rabbits treated with either irbesartan or rosuvastatin when compared with cholesterol diet group (Fig. 1). Rabbits treated with irbesartan showed no significant differences when compared with those treated with rosuvastatin for serum total cholesterol, triglyceride, LDL and VLDL levels with the exception that HDL levels in rosuvastatin-treated rabbits were significantly higher than those treated with Irbesartan (Fig. 1).

\section{Effect on Inflammatory Markers}

Data of this study have showed that serum hs-CRP, ICAM and VCAM levels were significantly increased in cholesterol diet group when compared with normal control group (Fig. 2a-c). Treatment of rabbits with either irbesartan or rosuvastatin significantly decreased serum hs-CRP, ICAM and VCAM levels in hyperlipidemic rabbits when compared with untreated cholesterol diet group (Fig. 2a-c). Rabbits treated with irbesartan showed no significant differences when compared with those treated with rosuvastatin for serum hs-CRP, ICAM and VCAM.

\section{Effect on Aortic Antioxidant Capacity}

After a 12-week challenge, aortic antioxidant capacity level was significantly decreased in cholesterol diet group (II) when

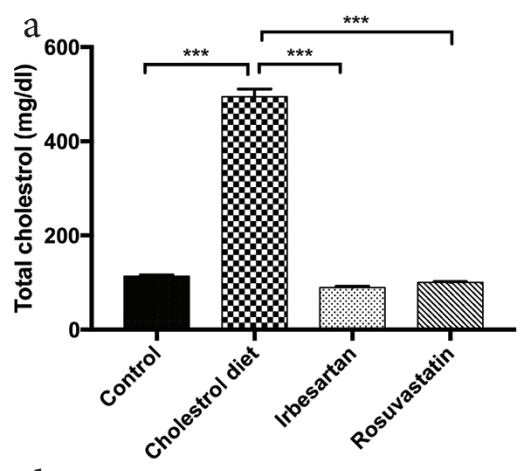

d

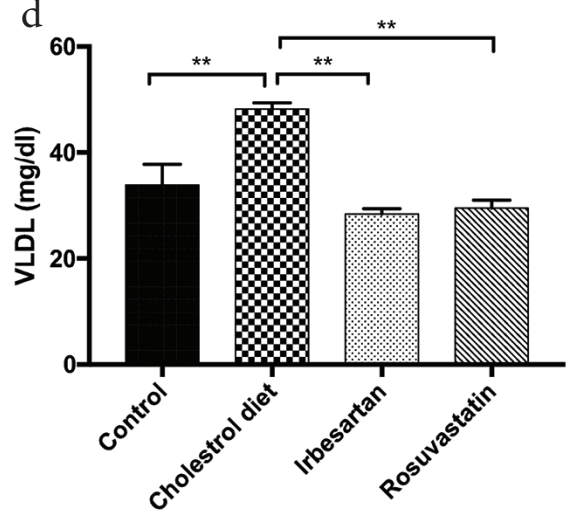

e
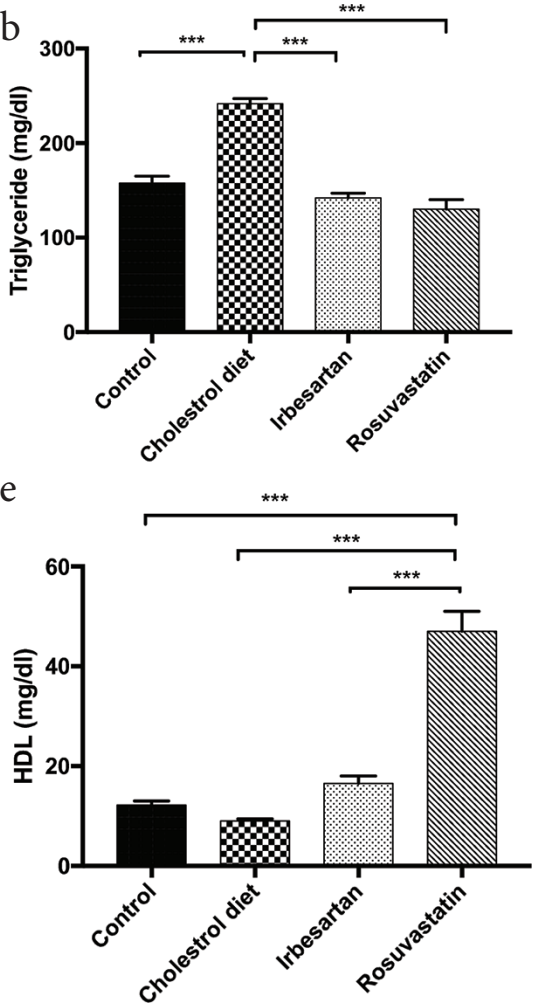

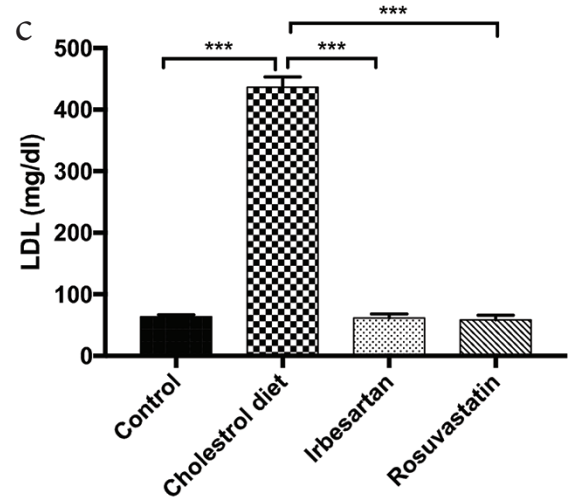

Fig. 1 Serum lipid profile (mg/dl). All data are presented as mean \pm SEM. **P $<0.01,{ }^{* * * P}<0.001$ 

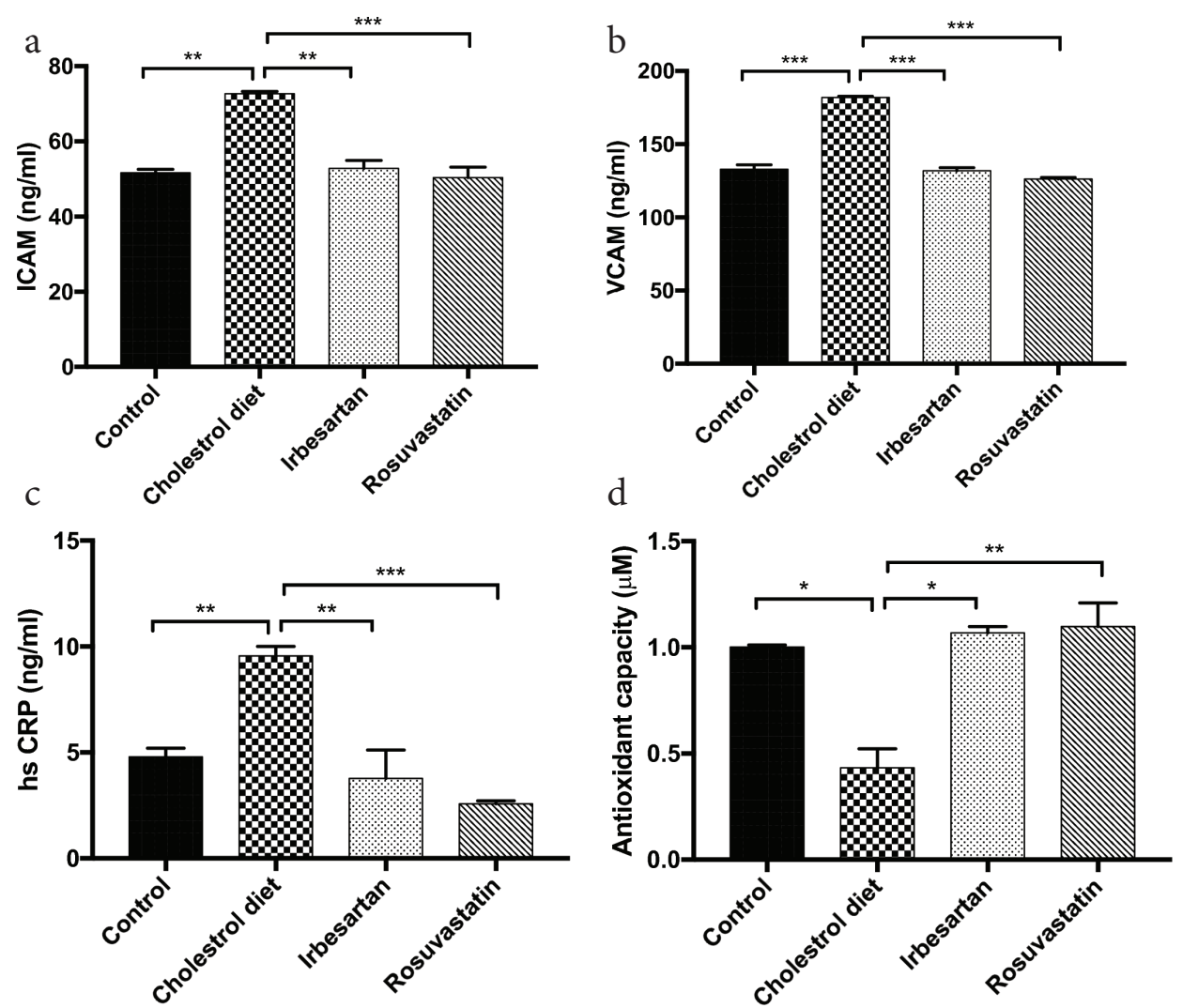

Fig. 2 Levels of ICAM, VCAM, hs-CRP and aortic antioxidant capacity. All data are presented as mean \pm SEM. $P<0.05$, $* * P<0.01, * * * P<0.001$.

compared with normal diet group (I) (Fig. 2d). However, treatments of rabbits with either irbesartan (group III) or rosuvastatin (group IV) significantly increased antioxidant capacity level when compared with untreated cholesterol diet group (Fig. 2d).

\section{Effect on Aortic Intima Media Thickness}

After a 12-week challenge, aortic intimal media thickness was significantly elevated $(P<0.05)$ in cholesterol diet group when compared with normal diet group (Table 1 and Figs. 3 and 4). However, treatments of rabbits with either irbesartan (group III) or rosuvastatin (group IV) significantly decreased aortic intimal media thickness $(P<0.05)$ when compared with untreated cholesterol diet group II (Table 1 and Figs. 5 and 6).

\section{Discussion}

Atherosclerosis is a major cause of ischemic heart disease that lead to myocardial infarction, angina and heart failure. Cholesterol and saturated fatty acid are the main contributing factor in exacerbation of atherosclerosis by building up of cholesterol in the wall of artery leading to narrowing down artery walls and this stricture limits oxygen supply to the site of organ especially heart. ${ }^{12,13}$

Table 1. Aortic intimal thickness (AIMT) changes in $\mu \mathrm{m}$

\begin{tabular}{lc}
\hline Study groups & AIMT $(\boldsymbol{\mu m})$ \\
\hline Normal diet group & $87.33 \pm 12$ \\
Cholesterol diet group & $325.83 \pm 12^{*}$ \\
Irbesartan group & $212.5 \pm 12^{* *}$ \\
Rosuvastatin group & $191.66 \pm 12^{* *}$ \\
\hline
\end{tabular}

${ }^{*} P<0.05$ is considered to be significant when compared with group I. ${ }^{* *} P<0.05$ is considered to be significant when compared with group II. All data are presented as mean \pm SEM

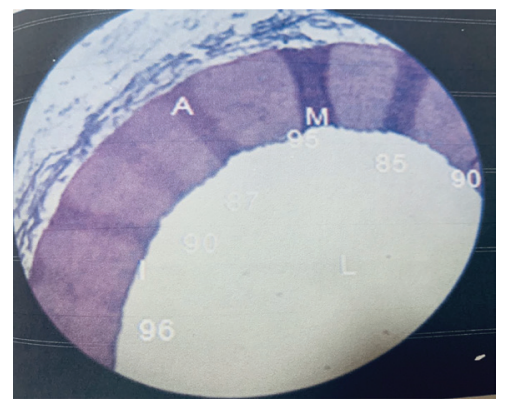

Fig. 3 Photomicrograph of histomorphometric section of aortic arch of rabbit (group I) normal control group that show intact endothelium with normal intimal thickness. The section stained with hematoxylin and eosin (10X). I: intima of the aorta, M: media of aorta, A: adventitia of aorta, L: lumen of aorta.

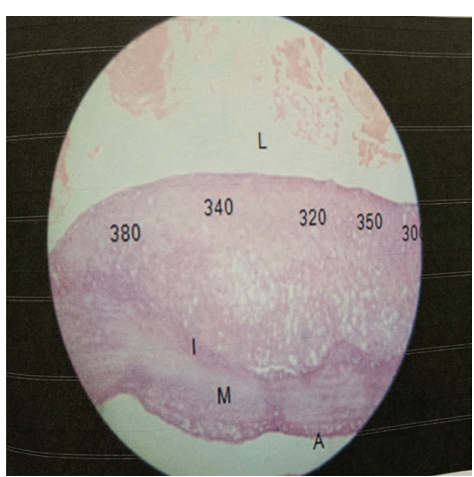

Fig. 4 Photomicrograph of histomorphometric section in aortic arch of rabbits on hypercholeterolemic diet group (II) for 12 weeks (induced untreated) show diffuse intimal thickening and incompletely coalesced extracellular lipid underneath a layers of macrophages and smooth muscle cells. The section stained with hematoxylin and eosin (10X). I: intima of the aorta, M: media of the aorta, A: adventitia of the aorta and L: the lumen of the aorta. 


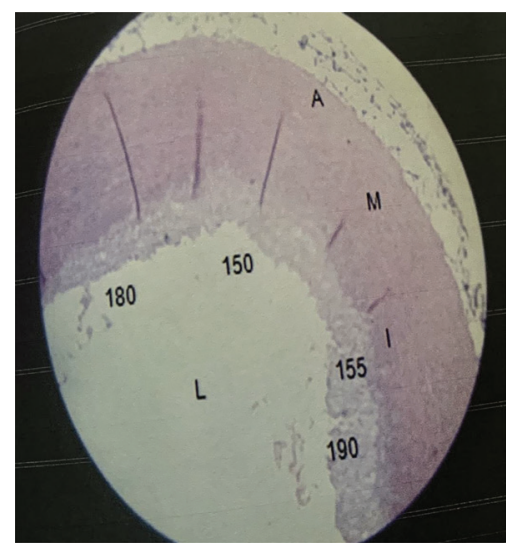

Fig. 5 Photomicrograph of histomorphometric section in aortic arch of rabbit treated with irbesartan (III). The section was stained with hematoxylin and eosin (10x). I: intima of the aorta, M: media of the aorta, A: adventitia of the aorta and $L$ : the lumen of the aorta.

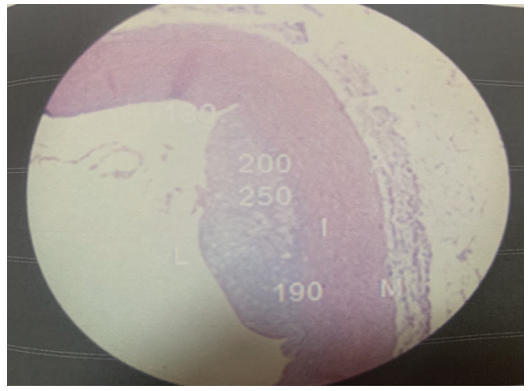

Fig. 6 Photomicrograph of histomorphometric section of aortic arch (group IV) treated with rosuvastatin that show layers of macrophage and smooth muscle cell, diffused intima thickening that incompletely coalesced. The section was stained with hematoxylin and eosin (10X). I: intima of the aorta, M: media of aorta, A: adventitia of aorta, L: lumen of aorta.

Data from this study have showed that a direct relationship between diet enriched with cholesterol and serum lipids profile (total cholesterol, TG, LDL, HDL, VLDL) is existed. Elevation of serum lipid profile has been noted in these groups after 12 weeks of cholesterol addition to diets and level of HDL decreased significantly, similar to the result by Oguntoye. ${ }^{12}$ Furthermore, a strong relationship between using of irbesartan treatment and decreasing of total cholesterol, TG, LDL, VLDL with little effect on HDL-C, where similar results have been found by Zhao et al. ${ }^{13}$ and Del Fiorentino et al. ${ }^{6}$ This could be ascribed to direct effect of peroxisome proliferation activated receptor alpha, this receptor regulates gene expression that is responsible for fatty acid oxidation. ${ }^{16}$

The renin-angiotensin-aldosterone system has been demonstrated to play not only an important role in cardiovascular homeostasis by influencing vascular tone and fluid-electrolyte balance, but has also been involved in the atherothrombotic process, cardiac remodeling and apoptosis. ${ }^{17}$

Angiotensin II, aldosterone, as well as endothelin-1 can modulate basal superoxide production by activation of reduced nicotinamide adenine dinucleotide phosphate (NADPH) oxidase and expression of its subunits. ${ }^{18}$ NADPH oxidase is the main source of reactive oxygen species (ROS) in the vascular wall, and is expressed in many cells including endothelial cells, vaso-active smooth muscle cells (VSMCs), fibroblasts and monocytes/macrophages. ${ }^{19,20}$ ROS act as signaling elements, but also in part by impairing endothelium-dependent vascular relaxation by reducing the bioavailability of nitric oxide (NO). ${ }^{21,22}$ Vascular contractile responses are accordingly enhanced, and this can be associated with structural changes in the wall of small arteries and increased peripheral vascular resistance..$^{23}$ Increased ROS production induced by angiotensin II is involved in the mechanisms leading to vascular remodeling, through VSMC proliferation and hypertrophy and collagen deposition..$^{24}$ ROS promote vascular inflammation and exert effects on the development and progression of atherosclerosis. ${ }^{25}$

Irbesartan was able to decrease hs-CRP, ICAM and VCAM. This is consistent with a study achieved by Liu et al., this decrease may be due to blockage of angiotensin II AT1 receptor resulting in inhibition of activation of NADPH oxidase which has a role in activation of nuclear factor kappa $B$. This transcription factor binds to promote region in the target gene leading to stimulation of transcription of pro-inflammatory cytokines mediators. ${ }^{26}$

Additionally, irbesartan has antioxidant activity may be due to blockage of AT1 receptor that inhibits activation of NADPH oxidase with a consequent inhibition of superoxide anion production, so decreases oxidative stress. ${ }^{27}$

In this study, irbesartan decreased aortic intimal media may be due to reducing the area of macrophages to total plaque. This decreases smooth muscle cell infiltration and reduce macrophage infiltration..$^{28}$

Finally, data from this study have showed that rosuvastatin was better than Irbesartan treated group in modulation of the parameters involved in atherosclerosis including lipid profile (TC, TG, LDL, VLDL and HDL), adhesion molecules and hs-CRP. Furthermore, rosuvastatin decreased aortic intimal media thickness by diminishing smooth muscle cell infiltration, connective tissue, macrophage accumulation and plaque disruption and by elevating collagen deposition in aorta. ${ }^{29}$

To conclude, irbesartan was able to retard progression of atherosclerosis in hypercholesterolemic male rabbits.

\section{Conflicts of Interest}

None.

\footnotetext{
References

1. Maria A, Yadav KS. Pathogenesis of atherosclerosis a review. Ann Clin Lab. 2016:4:2386-5180

2. de Paula Rogerio A, Sorgi C, Sadikot R, Carlo E. The role of lipids mediators in inflammation and resolution. BioMed Res Int. 2015;2015:1-2.

3. Munro JM, Cotran RS. The pathogenesis of atherosclerosis atherogenesis and inflammation. Lab Invest. 1988:58:249-261.
}

4. Dewberry R, Holden $H$, Crossman D, Francis S. Interleukin 1 receptor antagonist expression in human endothelium and atherosclerosis. Arterioscler Thromb Vasc Biol. 2000;20:2394-2400.

5. Sanz M, Ganado P. Tejerina T. Two angiotensin AT1 receptor antagonists, irbesartan and losartan, effects in cholesterol-fed rabbits. Eur J Pharmacol. 2002:442:99-106. 
6. Del Fiorentino A, Cianchetti S, Celi A, Dell'Omo G, Pedrinelli R. The effect of angiotensin receptor blockers on C-reactive protein and other circulating inflammatory indices in man. Vasc Health Risk Manag. 2009; 5: 233-242.

7. Blankenberg S, Barbaux S, Tiret L. Adhesion molecules and atherosclerosis. Atherosclerosis. 2003;170:191-123.

8. Preiss DJ, Sattar N. Vascular cell adhesion molecule-1: a viable therapeutic target for atherosclerosis. Int J Clin Pract. 2007;61:697-701.

9. Gumieniczek A. Effect of repaglinide on oxidative stress in tissue of diabetic rabbits. Diabetes Res Clin Pract. 2005;68:89-95.

10. Almeida EA, Hernandes BD, Ozaki MH, Ramírez-Núñez WR. Endothelium function, lipid peroxidation, plasmatic and tissue cholesterol evaluations in mixed dyslipidemia in rabbits treated with rosuvastatin and atorvastatin. Clín Invest Arterioscler. 2009;21:263-267.

11. Hietanen E, Aitio A, Koivusaari U, Kilpiö J, Nevalainen T, Närhi M, et al. Tissue concentrations and interaction of zinc with lead toxicity in rabbits. Toxicology 1982;25:113-127.

12. Oguntoye CO, Oke BO. A comparison of xylazine/ketamine, diazepam/ ketamine and acepromazine/ketamine anesthesia in rabbit. Sokoto J. Vet. Sci. 2014;12:21-25

13. Zhao Y, Fukao K, Zhao S, Watanabe A, Hamada T, Yamasaki K, et al. Irbesartan attenuates atherosclerosis in watanabe heritable hyperlipidemic rabbits: noninvasive imaging of inflammation by $18 \mathrm{~F}$-fluorodeoxyglucose positron emission tomography. Mol Imaging. 2015;14:1-8

14. Wong AP, Mohamed AL, Niedzwiecki A. Atherosclerosis and the cholesterol theory: a reappraisal. World J Cardiovasc Dis. 2016;6:391-409.

15. Liu T, Zhang L, Joo D, Sun SC. NF-KB signaling in inflammation. Signal Transduct Target Ther. 2017;2. pii: 17023.

16. Munger MA. Use of angiotensin receptor blockers in cardiovascular protection: current evidence and future directions. J Pharm Ther. 2011;36:22-40.

17. Biasucci LM, Lombardi M, Piro M, Di Giannuario G, Liuzzo G, Crea F. Irbesartan significantly reduces $C$ reactive protein concentrations after 1 month of treatment in unstable angina. Heart 2005;91:670-671.

18. Lassègue $B$, Clempus RE. Vascular NAD(P)H oxidases: specific features, expression, and regulation. Am J Physiol Regul Integr Comp Physiol. 2003;285:R277-R297.
19. Griendling KK, Sorescu D, Lassègue B, Ushio-Fukai M. Modulation of protein kinase activity and gene expression by reactive oxygen species and their role in vascular physiology and pathophysiology. Arterioscler Thromb Vasc Biol. 2000;20:2175-2183.

20. Touyz RM, Chen X, Tabet F, Yao G, He G, Quinn MT, et al. Expression of a functionally active gp91 phox-containing neutrophil-type NAD(P)H oxidase in smooth muscle cells from human resistance arteries: regulation by angiotensin II. Circ Res. 2002;90:1205-1213.

21. Rajagopalan S, Brook R, Mehta RH, Supiano M, Pitt B. Effect of losartan in aging related endothelial impairment. Am J Cardiol. 2002;89:562-566.

22. Jung O, Marklund SL, Geiger H, Pedrazzini T, Busse R, Brandes RP. Extracellular superoxide dismutase is a major determinant of nitric oxide bioavailability: in vivo and ex vivo evidence from ecSOD-deficient mice. Circ Res. 2003;93:622-629.

23. Touyz RM, Tabet F, Schiffrin EL. Redox-dependent signalling by angiotensin $\|$ and vascular remodelling in hypertension. Clin Exp Pharmacol Physiol. 2003;30:860-866.

24. Touyz RM, Schiffrin EL. Increased generation of superoxide by angiotensin II in smooth muscle cells from resistance arteries of hypertensive patients: role of phospholipase D-dependent NAD(P)H oxidase-sensitive pathways. J Hypertens. 2001;19:1245-1254.

25. Touyz RM, Schiffrin EL. Reactive oxygen species in vascular biology: implications in hypertension. Histochem Cell Biol. 2004;122:339-352.

26. Padoveze AF, Maniero F, Oliveira TV, Vitorio TS, Couto RD, Maranhão RC. Effect of a cholesterol-rich diet on the metabolism of the free and esterified cholesterol components of a Nano emulsion that resembles LDL in rabbits. Braz J Med Biol Res. 2009;42:172-178.

27. Li Z, Wang L, Hu X, Zhang P, Chen Y, Liu X, et al. Effect of rosuvastatin on atherosclerotic plaque stability: an intravascular ultrasound elastography study. Atherosclerosis 2016;248:27-35.

28. Zhang T, Shao B, Liu GA. Rosuvastatin promotes the differentiation of peripheral blood monocytes into M2 macrophages in patients with atherosclerosis by activating PPAR- $\gamma$. Eur Rev Med Pharmacol Sci. 2017;21:4464-4471. 